\title{
Extreme Nonlinear Optics in Terms of Riccati-Type Equation
}

\author{
R. PARzyński and A. Plucińska \\ Faculty of Physics, Adam Mickiewicz University \\ Umultowska 85, 61-614 Poznań, Poland
}

(Received July 21, 2005)

\begin{abstract}
In an approach based on the nonlinear Riccati-type differential equation for the ratio of the level population amplitudes, rather than on the optical Bloch equations, we describe the response of a two-level system to a few-cycle light pulse of intensity from the regime of extreme nonlinear optics, i.e., when the Rabi and transition frequencies are comparable. Emphasis is put on the dependence of the spectra of the scattered light on the carrier-envelope phase, duration and strength of both resonant and off-resonant pulses.
\end{abstract}

PACS numbers: 42.50.Md, 42.50.Hz, 42.65.Ky, 42.65.Re

\section{Introduction}

Recent experiments [1-3] on scattering of ultrashort (several femtoseconds), moderately intense (several $\mathrm{TW} / \mathrm{cm}^{2}$ ) light pulses by semiconductors ( $\mathrm{ZnO}, \mathrm{GaAs}$ ) have approached the regime of what is called extreme nonlinear optics. In the experiments, the optics has been extreme with respect to both the Rabi frequency reached, in fact comparable to the band-gap transition frequency due to large dipole transition moment, and pulse duration which was as short as about twice the optical period. Probably, the most spectacular observations were the spectral peaks in the scattered light occurring at the positions of even-order harmonics of the incident-pulse frequency and the dependence of these peaks on the phase of the carrier frequency with respect to the pulse envelope, the carrier-envelope phase. In the two-band approximation, the response of a semiconductor to a light pulse is known [4] to be described by the so-called semiconductor Bloch equations. In their mathematical structure, the semiconductor equations are identical with the familiar optical Bloch equations [5] for the two discrete-level system, but with the Rabi frequency modified additively by Coulombic interactions. Thus, emphasis has been put in [6] on solving (predominantly numerically and in part analytically) the optical Bloch equations in the extreme region without employing the conventional rotating-wave approximation (RWA) and with the neglect of trans- 
verse and longitudinal dampings, in fact, irrelevant on the time scale of few-cycle pulses. When light propagation effects could not be disregarded, the set of coupled Bloch-Maxwell equations has been solved numerically, instead. Interestingly, main features of the spectra produced from semiconductors have been successfully described in the framework of this fundamental two-level model of quantum and nonlinear optics. In the past, this model has also been used to describe nonlinear scattering of light by molecular ions [7-9] and double quantum wells [10, 11].

Appreciating the role the two-level models play in different branches of physics, we describe their optics, with particular emphasis on extreme nonlinear optics, in an alternative approach based on a single, nonlinear Riccati-type differential equation for the ratio of level population amplitudes. As a matter of fact, this equation takes its origin from the treatment of the magnetic resonance by Bloch and Siegert [12]. Adapted to the optical resonance [13], it was then solved analytically in a perturbative way to discuss non-RWA effects $[13,14]$. In the present paper we give an iterative algorithm solving the Riccati equation for arbitrary strengths, shapes, durations, and carrier-envelope phases of optical pulses. With this algorithm we study the evolutions of population inversion, induced dipole moment and electric field of scattered light as well as the corresponding spectra in the regime of extreme optics. A part of our spectra, obtained with the use of this algorithm, could be compared with those obtained from numerical integration of the optical Bloch equations and the available experimental data $[1-3,6]$. In this case, we have found general agreement. At a reasonably chosen time step the speed of our algorithm has been found competitive with that of the Mathematica package. The algorithm can also be used to solve the inverse problem, i.e., how to design the driving pulse to produce a desired population dynamics and spectra.

\section{The algorithm}

We apply the standard expansion, $\psi(t)=b_{1}(t)|1\rangle+b_{2}(t)|2\rangle$, for the wave function $\psi(t)$ of the system of two different-parity discrete levels of eigenfrequencies $\omega_{1}$ and $\omega_{2}>\omega_{1}$ in a pulsed laser field $\varepsilon(t)=\varepsilon_{0} h(t)$, where $h(t)=f(t) \cos \left(\omega_{0} t+\phi\right)$ describes the electric-field evolution with $0 \leq f(t) \leq 1$ having the sense of pulse envelope, $\omega_{0}$ the carrier frequency and $\phi$ the carrier-envelope phase. Without the RWA, the time-dependent population amplitudes, $b_{1}(t)$ and $b_{2}(t)$, are then to be found from the set of coupled linear equations, e.g. [5, 8]:

$$
\mathrm{i} \frac{\mathrm{d} b_{k}}{\mathrm{~d} t}=\omega_{k} b_{k}-\Omega(t) b_{l},
$$

where both $k$ and $l$ take the values 1,2 with the constraint $l \neq k$, and $\Omega(t)=$ $\Omega_{\mathrm{R}} h(t)$ is the instantaneous Rabi frequency with $\Omega_{\mathrm{R}}=\mu \varepsilon_{0} / \hbar$ being the usual Rabi frequency as determined by the electric-dipole transition matrix element, $\mu$, and the peak field value, $\varepsilon_{0}$. In the following we, however, reformulate the problem in terms of the ratio $r(t)=b_{2}(t) / b_{1}(t)$ of the population amplitudes. By 
differentiating $r$ over time, the set of two Eqs. (1) is known [12-15] to be replaced by a single equation for $r$, a nonlinear Riccati-type differential equation [16]:

$$
\mathrm{i} \frac{\mathrm{d} r}{\mathrm{~d} t}=\left(r^{2}-1\right) \Omega(t)+\omega_{21} r
$$

where $\omega_{21}=\omega_{2}-\omega_{1}$ is the transition frequency between the bare levels.

The last equation has no known general analytical solution covering the whole strength and time scales of the pulse. However, for an arbitrary strength, quite simple solutions are available for a sequence of short time intervals, each of duration $\Delta$ much shorter than the optical period $T=2 \pi / \omega_{0}$. Within, let us say, the $j$-th such an interval of the running time $(j-1) \Delta \leq t=t_{j} \leq j \Delta$, the field evolution function $h(t)$ can be considered nearly constant and approximated by $h_{j}=h\left(t=\left(j-\frac{1}{2}\right) \Delta\right)$, i.e., its value in the middle of the interval. The index $j$ runs the integers $1 \leq j \leq 2 N K$ for a $N$-cycle pulse whose half a cycle has been divided into $K$ equal-duration intervals $\Delta$. Due to the above approximation of piecewise constant field, the analytical solution of Eq. (2) at the end of the $j$-th interval, $r(t=j \Delta)=r_{j}$, turns out to be interrelated with that at the beginning of the $j$-th interval, $r(t=(j-1) \Delta)=r_{j-1}$, in the following way [17]:

$$
r_{j}=\frac{2 \Omega_{j}-\left[\omega_{21}+\mathrm{i} \Omega_{j}^{\mathrm{eff}} \cot \left(\Omega_{j}^{\mathrm{eff}} \Delta / 2\right)\right] r_{j-1}}{\omega_{21}-\mathrm{i} \Omega_{j}^{\mathrm{eff}} \cot \left(\Omega_{j}^{\mathrm{eff}} \Delta / 2\right)+2 \Omega_{j} r_{j-1}},
$$

where $\Omega_{j}=\Omega_{\mathrm{R}} h_{j}$ and $\Omega_{j}^{\mathrm{eff}}=\sqrt{4 \Omega_{j}^{2}+\omega_{21}^{2}}$.

We use Eq. (3) to formulate an iterative algorithm of solution of the general Eq. (2) and, thus, a description of the response of a two-level system to a strong, ultrashort laser pulse. Irrespective of the pulse strength, we can always choose the time interval $\Delta$ sufficiently short to satisfy the inequality $\Omega_{j}^{\text {eff }} \Delta / 2 \ll 1$ which, in practice, translates into $\Delta \ll 2 / \sqrt{4 \Omega_{\mathrm{R}}^{2}+\omega_{21}^{2}}$, equivalent to $K \gg \sqrt{4 x^{2}+y^{2}}$, where $x=\Omega_{\mathrm{R}} / \omega_{0}$ is a dimensionless strength parameter and $y=\omega_{21} / \omega_{0}$ a dimensionless level-separation parameter. With this choice, the cotangent function is approximated by its leading term converting Eq. (3) to its simplified version

$$
r_{j}=\frac{2 \Omega_{j} \Delta-\left(\omega_{21} \Delta+2 \mathrm{i}\right) r_{j-1}}{\omega_{21} \Delta-2 \mathrm{i}+2 \Omega_{j} \Delta r_{j-1}}=\frac{\frac{2 \pi x}{K} h_{j}-\left(\frac{\pi y}{K}+2 \mathrm{i}\right) r_{j-1}}{\frac{\pi y}{K}-2 \mathrm{i}+\frac{2 \pi x}{K} h_{j} r_{j-1}},
$$

where now the field evolution function $h(t)$ is expressed in terms of a dimensionless time parameter $\tau=\omega_{0} t$, meaning $h_{j}=h\left[\tau=\left(j-\frac{1}{2}\right) \frac{\pi}{K}\right]$. For fixed parameters $x$ and $y$, we establish $K$, reasonably, and then apply Eq. (4) to generate from the initial $r_{0}=b_{2}(0) / b_{1}(0)$ all successive $r_{j}$ for later points $j \frac{\pi}{K}$ of the time scale $\tau$. Equation (4) allows us to cover the time axis arbitrarily dense and thus to describe the dynamics of the system in any detail. In the present paper, we assume $r_{0}=0$ meaning that the lower level $|1\rangle$ is initially occupied.

From the found values of $r_{j}$ we straightforwardly calculate the population inversion, $w_{j}$, and the induced dipole moment, $d_{j}$, at successive discrete times $\tau=j \frac{\pi}{K}$ of the evolution. Since for any time, $w=\left|b_{2}\right|^{2}-\left|b_{1}\right|^{2}$ and $d=2 \mu \operatorname{Re}\left(b_{1}^{*} b_{2}\right)$ 
[5], we have from the population conservation law, $\left|b_{1}\right|^{2}+\left|b_{2}\right|^{2}=1$, and the definition of $r$ that

$$
w_{j}=\frac{\left|r_{j}\right|^{2}-1}{\left|r_{j}\right|^{2}+1}
$$

and

$$
d_{j}=2 \mu \frac{\operatorname{Re}\left(r_{j}\right)}{\left|r_{j}\right|^{2}+1} .
$$

Since for a two-level system, the induced dipole moment satisfies the equation $\ddot{d}=-\omega_{21}^{2} d-2 \mu \omega_{21} \Omega(t) w[18]$, the evolution of the electric field produced by this dipole can be calculated from

$$
\begin{aligned}
\varepsilon_{j}^{\mathrm{sc}} & =\varepsilon^{\mathrm{sc}}\left(\tau=j \frac{\pi}{K}\right) \sim-y^{2} d_{j}-2 \mu y x h_{j} w_{j} \\
& =\frac{-2 \mu y}{\left|r_{j}\right|^{2}+1}\left[y \operatorname{Re}\left(r_{j}\right)+x h_{j}\left(\left|r_{j}\right|^{2}-1\right)\right] .
\end{aligned}
$$

Finally, to a given discrete function $g_{j}=g(\tau=j \pi / K)$ one can assign its discrete Fourier transform

$$
G(z)=\frac{1}{\sqrt{n}} \sum_{j=1}^{n} g_{j} \exp [2 \pi \mathrm{i}(j-1)(z-1) / n],
$$

where in our case $n=2 N K$. Equation (8) determines the frequency spectrum, $|G(z)|^{2}$, of the time-dependent function $g$.

\section{Applications}

We now apply the above procedure, primarily based on the algorithm of Eq. (4), to the regime of extreme optics. The pulse parameters to be chosen are inspired by the recent experiments $[1,2]$, real or/and numerical ones, in which a few-cycle optical pulses were applied to different semiconductor materials modeled by two bands.

\subsection{Off-resonant excitation}

In the off-resonant experiment by Tritschler et al. [1] on $\mathrm{ZnO}$ semiconductor $(\mu=0.19 e \mathrm{~nm}=9.12 \mathrm{D})$, the applied pulse strength $\left(\varepsilon_{0}=6 \times 10^{9} \mathrm{~V} / \mathrm{m} \rightarrow\right.$ $5 \mathrm{TW} / \mathrm{cm}^{2}=1.42 \times 10^{-4}$ a.u. of intensity) and frequency $\left(\hbar \omega_{0}=1.5 \mathrm{eV}\right.$, half of the band gap energy) translate into our $x=0.76$ and $y=2$ parameters. We approximate the pulse shape as $f(\tau)=\operatorname{sinc}\left[\left(\tau-\frac{1}{2} \tau_{\max }\right) / \tau_{0}\right]$, where $\operatorname{sinc}(x)=$ $\sin (x) / x, \tau_{0}$ is related to the full width at half maximum (FWHM) of intensity by $\tau_{0}=\tau_{\mathrm{FWHM}} / 2.7831$, and the scaled running time $0 \leq \tau \leq \tau_{\max }$ does not exceed $\tau_{\text {max }}=2 \pi \tau_{0}=2.257 \tau_{\mathrm{FWHM}}$ ensuring $f(\tau)$ to be positive at any point of the time scale. From now on we write $\tau_{\mathrm{FWHM}}=2 \pi N_{\mathrm{FWHM}}$ with $N_{\mathrm{FWHM}}$ meaning the width in units of the optical period $T=2 \pi / \omega_{0}=2.76 \mathrm{fs}$. We adopt the experimental [1] $\mathrm{FWHM}=5$ fs corresponding to $N_{\mathrm{FWHM}}=1.81$ and, additionally, assume the carrier-envelope phase $\phi=0$. For this incident pulse we show in Fig. 1 some results obtained from Eqs. (4)-(8) with $K=200$ satisfying the required condition 

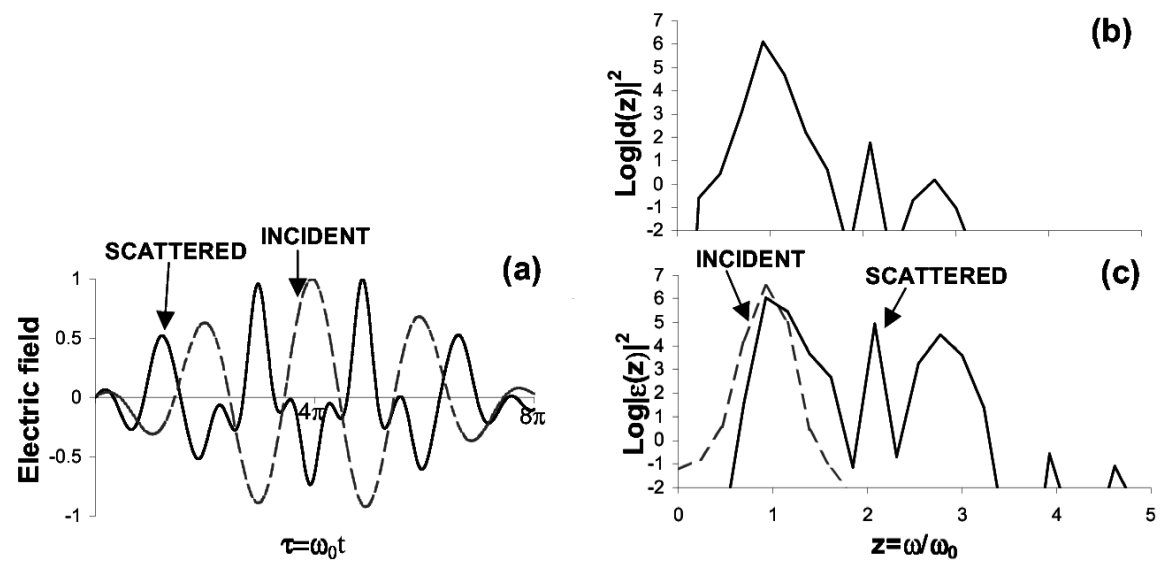

Fig. 1. (a) Evolution of the scattered field (solid line) in comparison to that of the incident field (dashed line), (b) spectrum of the induced dipole, (c) spectrum of the scattered light (solid line) compared to that of the incident light (dashed line). All curves have been obtained for the $h(\tau)=\operatorname{sinc}\left[\left(\tau-\frac{1}{2} \tau_{\max }\right) / \tau_{0}\right] \cos \tau$ incident pulse of $\tau_{0}=$ $\tau_{\text {FWHM }} / 2.7831, \tau_{\text {FWHM }}=2 \pi N_{\text {FWHM }}, N_{\text {FWHM }}=1.81, \tau_{\max }=2 \pi \tau_{0}$ and the strength-frequency parameters $x=0.76$ and $y=2$ (off-resonant excitation). In (a), the curves are normalized to the same height. In (a), (b) and (c), the vertical axes are scaled in relative units.

$K \gg \sqrt{4 x^{2}+y^{2}}$. However, we have found that for $K=50$, i.e. diminished by the factor of 4 , the obtained results remain the same, but required computer time is shortened substantially. As seen from Fig. 1a, the calculated time evolution of the scattered field (solid line) differs markedly from that of the incident field (dashed line). The same concerns the frequency spectrum found from Eq. (8). In Fig. $1 \mathrm{~b}$ we show the power spectrum of the induced dipole moment, $|d(z)|^{2}$, and in Fig. 1c the power spectra, $|\varepsilon(z)|^{2}$, of the scattered (solid line) and incident (dashed line) fields. Though differing in details, the $|d(z)|^{2}$ and $|\varepsilon(z)|^{2}$ spectra give qualitatively the same information on the frequency components included. The most interesting result is the peak at the position of the second $\left(z=\omega / \omega_{0} \approx 2\right)$ harmonic of the pulse frequency $\omega_{0}$, appearing due to interference of tails of the neighboring spectrally broad odd components. The peak at $z \approx 2$ has been given a lot of attention by Tritschler et al. [1], who named it the third harmonic in disguise of the second harmonic. The height of our peak at $z \approx 2$, in relation to the heights of the neighboring peaks, nearly agrees with the result of the numerical experiment of Tritschler et al. (Fig. 1 in [1]) exploiting the optical Bloch equations, though a slightly different pulse envelope was used in the experiment (the long-time tails of the $\sin (x) / x$ function were suppressed by a proper Gaussian).

The peak at $z \approx 2$, as being an effect of the broad spectrum of the incident pulse, vanishes with increasing duration of the pulse. This is confirmed by Fig. 2 showing the calculated power spectrum of the induced dipole versus integer mul- 

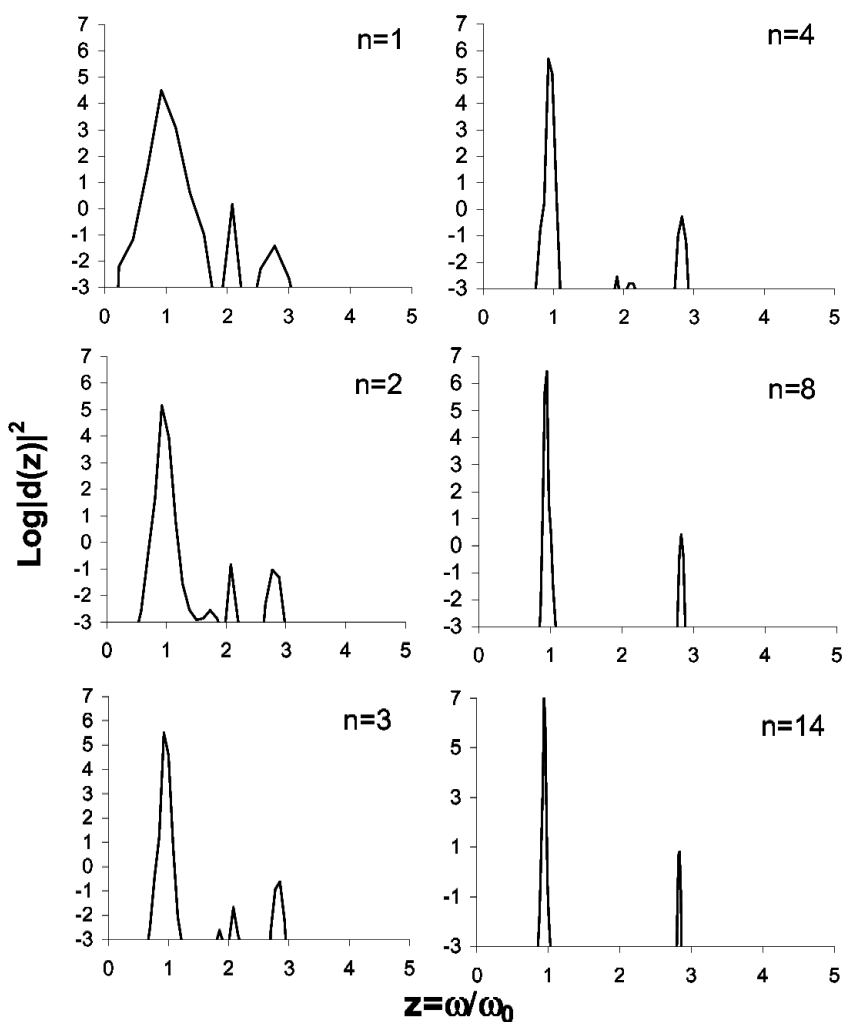

Fig. 2. Evolution of the spectrum from Fig. 1b with increasing pulse duration $N_{\text {FWHM }}=1.81 n(n=1,2,3,4,8$ and 14$)$ measured in units of optical period of the incident light.

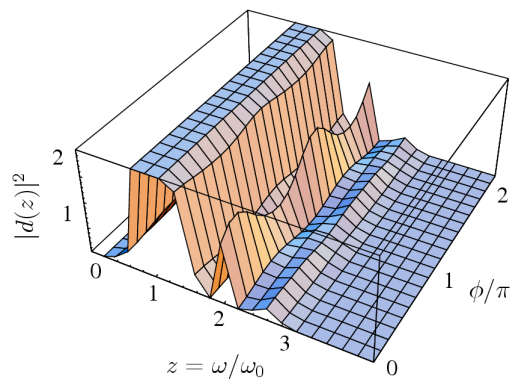

Fig. 3. Effect of carrier-envelope phase $\phi$ on the spectrum from Fig. 1b. The peak around $z=1$ has been suppressed by a factor of $10^{4}$ to include it in the figure.

tiplicity of the originally assumed pulse length $\left(|d(z)|^{2}\right.$ versus $N_{\mathrm{FWHM}}=1.81 n$; $n=1,2,3,4,8,14)$. In general, the increase in the pulse length results in increasing the fundamental and third-harmonic peaks and decreasing the peak around $z \approx 2$. 
As seen, the increase in the pulse length by a factor of $n=8$ results in practically complete suppression of the peak around $z \approx 2$. This figure clearly shows that the peak around $z \approx 2$ is a few-cycle effect and vanishes in the conventional regime of many-cycle pulses.

Due to its interference origin, the peak around $z \approx 2$, particularly well pronounced when $N_{\text {FWHM }}=1.81$, exhibits sensitivity to the carrier-envelope phase $\phi$. The calculated oscillatory $\phi$-dependence with the period of $\pi$ is shown in Fig. 3 .

\subsection{Resonant excitation}

For the pulse shape as previously and $\phi=0$, we change the parameters into $x=1.236, y=1$ and $N_{\mathrm{FWHM}}=1.92$, which correspond to the conditions under which Mücke et al. [2] have integrated numerically the coupled Maxwell-Bloch equations for the GaAs semiconductor $\left(\mu=0.5 \mathrm{e} \mathrm{nm}=24 \mathrm{D}, \varepsilon_{0}=3.5 \times 10^{9} \mathrm{~V} / \mathrm{m} \rightarrow\right.$ $1.7 \mathrm{TW} / \mathrm{cm}^{2}=4.8 \times 10^{-5}$ a.u. of intensity, $\hbar \omega_{0}=1.42 \mathrm{eV} \rightarrow T=2.92 \mathrm{fs}$, FWHM $=5.6 \mathrm{fs})$. Due to the band-gap resonance $(y=1)$ the dynamics of the system is now quite different. Some striking differences, calculated from our algorithm (Eq. (4)) with $K=200$, are shown in Fig. 4, where we compare the evolutions of inversion and those of induced dipole for two cases, the present resonant $\left(y=1, x=1.236, N_{\mathrm{FWHM}}=1.92\right)$ and the previous off-resonant $\left(y=2, x=0.76, N_{\mathrm{FWHM}}=1.81\right)$ cases. The off-resonant pulse moves instantaneously only a small part (0.175 at most) of the initial population towards the higher state, while the resonant pulse moves the whole population from the lower state via the upper state back to the lower state three times. These three

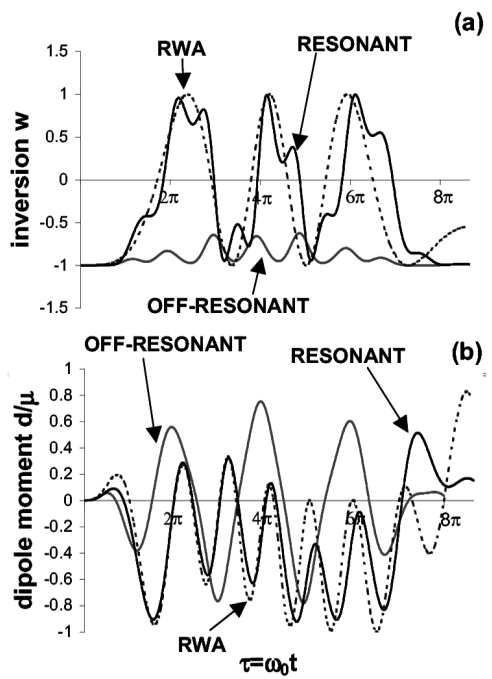

Fig. 4. Evolution of population inversion, (a), and that of induced dipole, (b), for resonant $\left(y=1, x=1.236, N_{\mathrm{FWHM}}=1.92\right)$ and off-resonant $(y=2, x=0.76$, $\left.N_{\text {FWhm }}=1.81\right)$ excitations. The dashed curves are the results of the RWA for the resonant excitation. 
Rabi oscillations are roughly consistent with the calculated pulse area

$$
A\left(\tau=\tau_{\max }\right)=x \int_{0}^{\tau_{\max }} f(\tau) \mathrm{d} \tau=2 x \tau_{0} \int_{0}^{\pi} \operatorname{sinc}(u) \mathrm{d} u \approx 3.7 x \tau_{0} \approx 3.16(2 \pi) .
$$

However, the actual evolution of the inversion by no means satisfies the familiar $w=-\cos [A(\tau)]$ law [5] because the strength parameter $x$ is well beyond the rotating-wave approximation requiring $x \ll 1$. For the same reason, the actual induced dipole moment does not follow the $d=\mu \sin [A(\tau)] \sin (\tau)$ law of the rotating-wave approximation for a resonant frequency [5].

As a result, the spectra are qualitatively different in the resonant case. In Fig. 5 we show the spectrum of scattered light, $\left|\varepsilon^{\mathrm{sc}}(z)\right|^{2}$, which is composed of three broad peaks at the positions of even harmonics $(z \approx 2,4,6)$. These peaks exhibit the dependence on the carrier-envelope phase $\phi$ with the period of $\pi$ (Fig. 6). The $\phi$-dependence of the peak at $z \approx 2$ can be compared with the result of numerical integration of the Maxwell-Bloch equations by Mücke et al. (Fig. 2b in [2]) and we find agreement between the two results. As shown in Fig. 7, the peaks do not vanish with increasing pulse duration, they get even more pronounced and separated for longer pulses $\left(N_{\mathrm{FWHM}}=10 \cdot 1.92\right)$. The qualitative differences in the spectrum originate from a different mechanism of peak formation. In the resonant case, it is predominantly the interference of different Mollow side bands of two adjacent odd harmonics [6]. Since the creation of the Mollow side bands

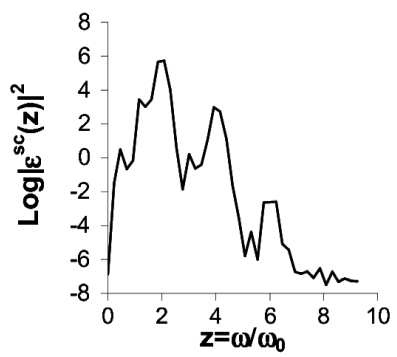

Fig. 5. Spectrum of the scattered light in the case of resonant excitation $(y=1$, $\left.x=1.236, N_{\mathrm{FWHM}}=1.92\right)$.
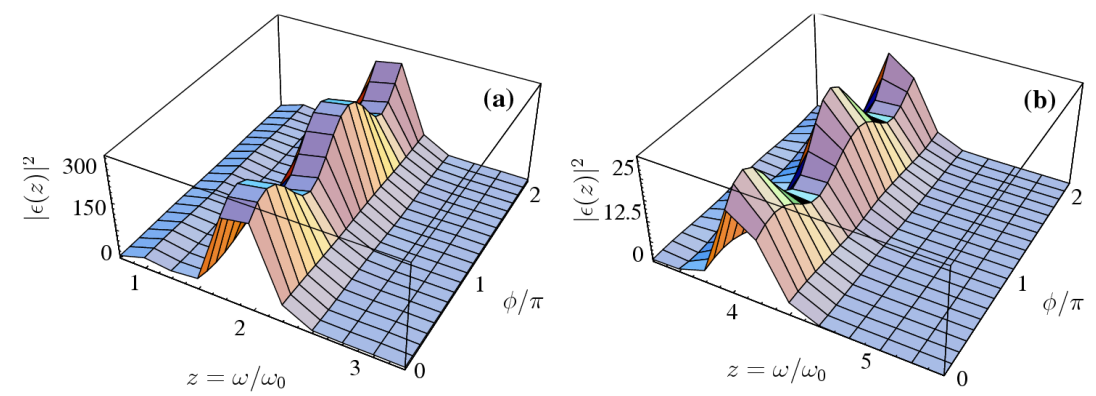

Fig. 6. Dependence of the spectrum around $z=2$ and $z=4$ from Fig. 5 on the carrier-envelope phase $\phi$. 


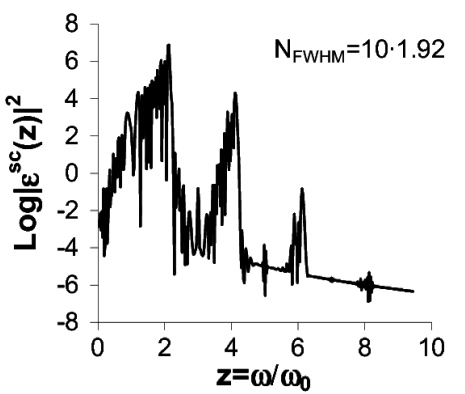

Fig. 7. As in Fig. 5 but for a longer pulse of $N_{\mathrm{FWHM}}=10 \cdot 1.92$.
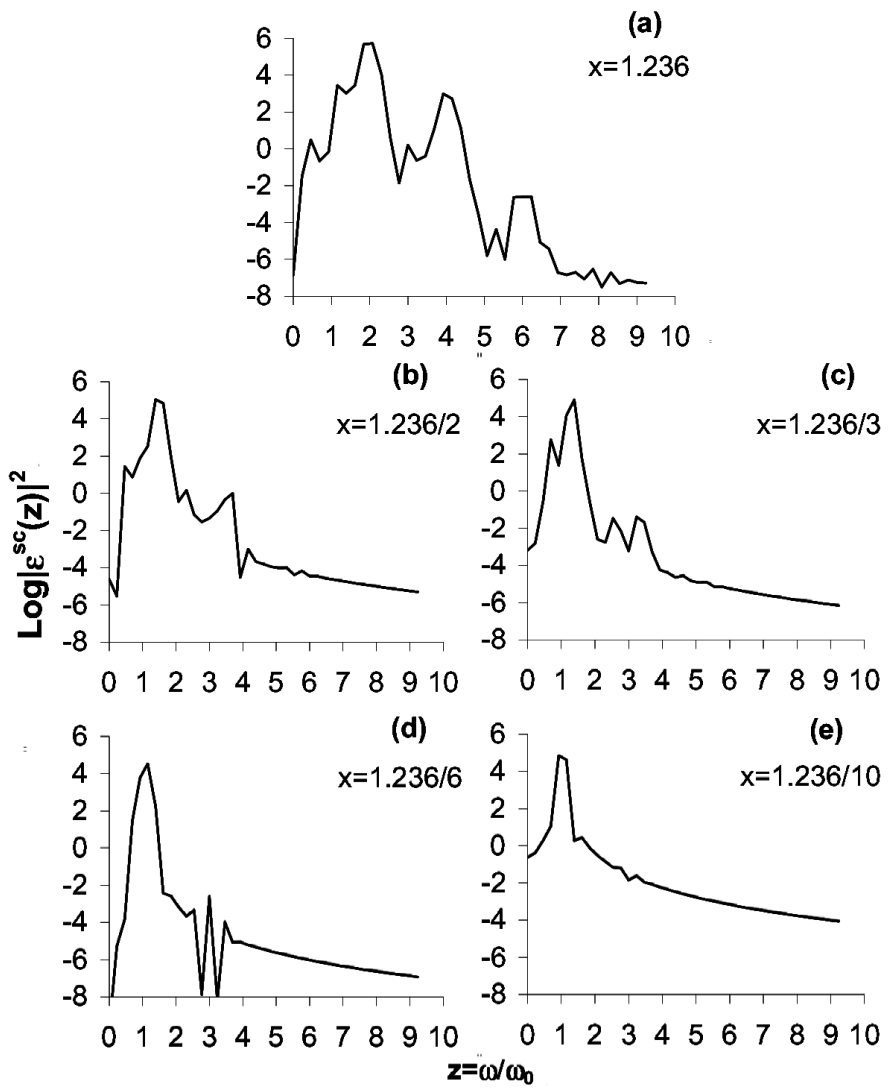

Fig. 8. As in Fig. 5 but for weaker pulses.

requires the appropriate pulse strengths, the spectrum from Fig. 5 changes its character when varying $x$. In Fig. 8 we show the conversion of the even- $z$ peaks into odd- $z$ peaks when making $x$ smaller. The behavior of the spectrum around $z=3$, shown in Fig. $8 \mathrm{~b}-\mathrm{d}$, is in qualitative agreement with that observed in a 
different resonant $(y=1)$ experiment of Mücke et al. [3] in which the 5 fs pulse of the hyperbolic secant envelope $\left(f(\tau)=\operatorname{sech}\left(\tau / \tau_{0}\right), \tau_{0}=\tau_{\mathrm{FWHM}} / 1.763\right)$ has produced the third harmonic peak from the GaAs semiconductor and converted this peak into a doublet when increasing, in some range, the pulse area $A$ related to our parameter $x$ through $A=\pi \tau_{0} x$.

\section{Summary}

In the form of Eq. (4) we have given an iterative algorithm for solving the Riccati-type equation for the ratio of population amplitudes of a two-level system in a pulsed oscillating field. The approach based on the Riccati-type equation $[12-15,17]$ is an alternative to the commonly used one exploiting the optical Bloch equations $[1-7,10,11,19-21]$. Our algorithm suffers no approximations, like the rotating-wave [5] and adiabatic [18] approximations. Applied to the regime of extreme optics, the algorithm has provided the results on the population inversion in the system, polarization of the system and spectra of scattered light versus different parameters of the incident pulse like the central frequency, duration, strength and carrier-envelope phase. A part of the results could be compared with success with the existing experimental results or the results obtained by others from integrating numerically the optical Bloch equations.

As a test of efficiency, we have compared the times consumed by a standard personal computer when finding the evolutions of population inversion and induced dipole from our algorithm and from the optical Bloch equations $(\mathrm{d} u / \mathrm{d} \tau=y v$, $\mathrm{d} v / \mathrm{d} \tau=-y u-2 x h(\tau) w$ and $\mathrm{d} w / \mathrm{d} \tau=2 x h(\tau) v$, in our terminology) solved by the appropriate Mathematica package. For the very dense graining of the time axis ( $K=200 \gg \sqrt{4 x^{2}+y^{2}}$ at the $x, y$ parameters of this paper), our algorithm was found to be less efficient by approximately a factor of 5 . In practice, this factor is not substantial in a few-cycle regime, where the overall computer-time consumed is of the order of several seconds. However, at moderate graining $(K=50)$ the results were obtained in a flash and the speed of our algorithm was found competitive with that offered by the package Mathematica. Such a diminishing of $K$ has not introduced any change to the final results, because $K$ still satisfies the relation $K \gg \sqrt{4 x^{2}+y^{2}}$. An additional advantage of our algorithm is that it offers a way for solving the inverse problem consisting in designing the driving pulse $\varepsilon(t)=\varepsilon_{0} h(\tau)$ that will cause a desired population dynamics $(r(\tau))$ and related spectra. Indeed, Eq. (4) allows to find $x h_{j}$ from the fixed values of $r_{j-1}$ and $r_{j}$. We have checked that, for the $x, y$ parameters of this paper, the inversion procedure works well.

\section{References}

[1] T. Tritschler, O.D. Mücke, M. Wegener, U. Morgner, F.X. Kärtner, Phys. Rev. Lett. 90, 217404 (2003).

[2] O.D. Mücke, T. Tritschler, M. Wegener, U. Morgner, F.X. Kärtner, Phys. Rev. Lett. 89, 127401 (2002). 
[3] O.D. Mücke, T. Tritschler, M. Wegener, U. Morgner, F.X. Kärtner, Phys. Rev. Lett. 87, 057401 (2001).

[4] H. Haug, S.W. Koch, Quantum Theory of the Optical and Electronic Properties of Semiconductors, World Scientific, Singapore 1990, Chs. 5, 10 and 12.

[5] L. Allen, J.H. Eberly, Optical Resonance and Two-Level Atoms, Dover Publications, New York 1987.

[6] T. Tritschler, O.D. Mücke, M. Wegener, Phys. Rev. A 68, 033404 (2003).

[7] T. Zuo, S. Chelkowski, A.D. Bandrauk, Phys. Rev. A 48, 3837 (1993); Phys. Rev. A 49, 3943 (1994).

[8] M.Yu. Ivanov, P.B. Corkum, Phys. Rev. A 48, 580 (1993).

[9] M.Yu. Ivanov, P.B. Corkum, P. Dietrich, Laser Phys. 3, 375 (1993).

[10] R. Bavli, H. Metiu, Phys. Rev. Lett. 69, 1986 (1992); Phys. Rev. A 48, 886 (1993); Phys. Rev. A 47, 3299 (1993).

[11] A. Levinson, M. Segev, G. Almogy, A. Yariv, Phys. Rev A 49, 661 (1994).

[12] F. Bloch, A. Siegert, Phys. Rev. 57, 522 (1940).

[13] G.M. Genkin, Phys. Rev. A 58, 758 (1998).

[14] R. Parzyński, M. Sobczak, Opt. Commun. 228, 111 (2003); J. Phys. B, At. Mol. Opt. Phys. 37, 743 (2004).

[15] D.E. Rourke, L. Khodarinova, A.A. Karabanov, Phys. Rev. Lett. 92, 163003 (2004).

[16] D. Zwillinger, Handbook of Differential Equations, Academic Press, New York 1998.

[17] R. Parzyński, M. Sobczak, A. Plucińska, Acta Phys. Pol. A 106, 827 (2004).

[18] L. Plaja, L. Roso-Franco, J. Opt. Soc. Am. B 9, 2210 (1992).

[19] S. Hughes, Phys. Rev. Lett. 81, 3363 (1998); Phys. Rev. A 62, 055401 (2000).

[20] V.P. Kalosha, J. Herrmann, Phys. Rev. Lett. 83, 544 (1999).

[21] B. Sundaram, P.W. Milonni, Phys. Rev. A 41, 6571 (1990). 